\title{
T Cell Receptor Biases and Clonal Proliferations among Lung Transplant Recipients with Obliterative Bronchiolitis
}

\author{
Steven R. Duncan, ${ }^{\star}$ Vincent Valentine, ${ }^{\S}$ Mihovil Roglic, ${ }^{\star}$ Darlene J. Elias, ${ }^{\ddagger}$ Katherine W. Pekny, ${ }^{\star}$ James Theodore, ${ }^{\S}$ \\ Dwight H. Kono, ${ }^{\star}$ and Argyrios N. Theofilopoulos* \\ Departments of *Immunology and ${ }^{\ddagger}$ Experimental Medicine, Scripps Research Institute, La Jolla, California 92037 ; and ${ }^{\S}$ Department of \\ Medicine, Stanford University Medical Center, Stanford, California 94305
}

\begin{abstract}
Obliterative bronchiolitis $(\mathrm{OB})$ is the most serious late complication of lung transplantation, but the pathogenesis of this disorder has not been elucidated. We sought evidence that $\mathrm{OB}$ is mediated by a cellular immunologic response by characterizing $T$ cell antigen receptor $\beta$-chain variable gene (TCRBV) repertoires in lung allograft recipients. Expression levels of 27 TCRBV among recipients were determined by multiprobe RNase protection assay after PCR amplification. In comparison to recipients with no evidence of rejection $(n=9)$, the PBL TCRBV repertoires of OB subjects $(n=16)$ exhibited more frequent expansions (16 vs. $9 \%$ of all measured TCRBV, $P<0.02$ ), and the magnitudes of these abnormalities were greater $(8.2 \pm 0.8$ vs. $4.5 \pm 0.3 \mathrm{SD}$ from mean normal values, $P<0.01)$. TCRBV sequencing showed these expansions were composed of clonal or oligoclonal populations. Thus, $T$ cell responses in the recipients are marked by highly selective clonal expansions, presumably driven by indirect recognition of a limited number of immunodominant alloantigens. These processes are exaggerated among allograft recipients with $\mathrm{OB}$, implying that cognate immune mechanisms are important in the pathogenesis of the disorder. Furthermore, the prominence of finite, distinct TCR phenotypes raise possibilities for development of novel diagnostic modalities and targeted immunotherapies for OB and other manifestations of chronic allograft rejection. (J. Clin. Invest. 1996. 97:2642-2650.) Key words: allograft rejection, chronic - T cell antigen receptors, $\alpha \beta \cdot$ organ transplantation
\end{abstract}

\section{Introduction}

The long-term functional status and survival of lung transplant recipients are often compromised by development of obliterative bronchiolitis (OB), ${ }^{1}$ a fibroproliferative injury of distal air-

Address correspondence to A.N. Theofilopoulos, M.D., Department of Immunology, IMM3, 10666 N. Torrey Pines Road, L Jolla, CA 92037. Phone: 619-554-8135; FAX: 619-554-6229.

Received for publication 9 January 1996 and accepted in revised form 14 March 1996.

1. Abbreviations used in this paper: $\mathrm{BAL}$, bronchoalveolar lavage; $\mathrm{BC}, \beta$-chain constant region; $\mathrm{BV}, \beta$-chain variable region; NER, no evidence of rejection; OB, obliterative bronchiolitis; TCR, T cell antigen receptor; VDJ, variable, diversity, joining segments.

J. Clin. Invest.

(C) The American Society for Clinical Investigation, Inc.

0021-9738/96/06/2642/09 \$2.00

Volume 97, Number 11, June 1996, 2642-2650 ways and small pulmonary blood vessels. OB occurs in as many as $50 \%$ of pulmonary allograft recipients and, while the severity varies widely, typically leads to progressive airflow obstruction, as well as increased risks for infections and mortality $(1,2)$. Although OB is generally acknowledged to be a manifestation of chronic allograft rejection, the underlying immunopathogenesis of the disorder remains largely speculative (3).

An approach to understanding $\mathrm{T}$ cell responses at the molecular level has been afforded by the recent development of methodologies that enable characterization of $\mathrm{T}$ cell antigen receptor (TCR) $\alpha$ and $\beta$ chain gene expression among cell populations that have been selectively expanded or deleted by interactions with antigens $(4,5)$. An enormous potential range of receptor specificities for antigenic epitopes is generated during lymphocyte ontogeny by random combinatorial rearrangements of ubiquitous genes encoding variable (V), diversity (D) ( $\beta$ chain only), and joining ( $\mathrm{J}$ ) segments. The distinctiveness of individual TCR repertoires is shaped by intrathymic positive and negative selections for self-antigens and, ultimately, effects of receptor engagements with exogenous ligands. Depending on the nature of the stimulus, these peripheral TCRantigen interactions may, in turn, result in clonal proliferation, anergy, or deletion of the engaged T cells (6). TCR expression in human diseases has been most extensively studied in various autoimmune disorders (4-6). In comparison, the influences of alloantigen engagements on TCR profiles among human transplant recipients have received relatively little investigation.

A more complete delineation of TCR repertoires in transplant recipients could lead to fundamental insights into the nature of T cell-alloantigen interactions and the pathogenesis of rejection phenomenon, and may eventually have clinical importance with respect to devising more effective therapeutic modalities (3, 7-10). Accordingly, we undertook these studies using a novel methodology to quantitate TCR $\beta$-chain variable region (TCRBV) gene expressions in lymphocytes of peripheral blood (PBL) and bronchoalveolar lavage (BAL) from lung transplant recipients. The study of in vivo allogeneic responses is facilitated in this population by the ease with which infiltrating cells can be harvested from large anatomical areas of the allograft (i.e., by BAL). Furthermore, the histologic and functional status of the allograft can be serially ascertained by biopsy and spirometry, respectively. Our findings suggest that cognate cellular immune responses play an important role in the development of $\mathrm{OB}$ and that these responses are characterized by oligoclonal proliferations driven by a limited number of immunodominant alloepitopes.

\section{Methods}

Patient specimens. Lung transplant recipients were recruited during the course of their routine clinical evaluations. Normal subjects were 
recruited by advertisement. All gave informed consent under the auspices of the appropriate Institutional Review Board. Inclusion criteria for recipients consisted of an unequivocal diagnosis of either OB or no evidence of rejection (NER) established by histologic evaluations of surveillance lung biopsies using standardized criteria (11), and elapsed time since transplantation $>12 \mathrm{wk}$. Transplant recipients were maintained on stable doses of immunosuppressants (e.g., cyclosporine A, azathioprine, low-dose glucocorticosteroids) as detailed elsewhere $(1,2)$. Subjects were excluded if they had evidence of infections or received treatments that could transiently alter $\mathrm{T}$ cell subsets (e.g., augmented immunosuppressants, antithymocyte globulin) within the preceding $8 \mathrm{wk}$.

Mononuclear leukocytes were separated from 20 to $50 \mathrm{ml}$ of blood by density gradient centrifugation. Cell subpopulations were delineated by direct staining with fluorochrome-conjugated monoclonal antibodies against CD3, CD4, CD8, and MHC Class II DR (Pharmingen, San Diego, CA), and analyzed by FACS. BAL was performed in transplant recipients when clinically indicated using four $50-\mathrm{ml}$ aliquots of saline in the right middle lobe or lingular bronchi. A portion $(40-50 \mathrm{ml})$ of the return from the co-mixed second and subsequent aliquots was processed as described for PBL.

Reverse transcription $-P C R$. This methodology was developed to enable TCR repertoire determinations from specimens with small numbers of cells, while precluding quantitative inaccuracies due to differential PCR primer-template binding/extension efficacies (3).
Total cellular RNA was extracted from specimens by guanidinium thiocyanate isolation (12). First strand cDNA was synthesized using Moloney MuLV reverse transcriptase (Boehringer Mannheim Biochemicals, Indianapolis, IN) with oligo-dT primers (Fig. 1). dG-tails were added to the cDNA by terminal deoxynucleotidyl transferase using $500 \mu \mathrm{M}$ dGTP. Purified product (GeneClean; Bio 101, La Jolla, CA) was subjected to PCR amplification using Taq polymerase, following the GeneAmp protocol (Perkin-Elmer-Cetus, Emeryville, CA). A $50 \mu l$ reaction using a primer to the TCR $\beta$-chain constant region (BC) (5'CGAGGTAAGCCACAGTCTGCTCTA), as well as T7-C (5'GCCAGTGAATTGTAATACGACTCACTATAGGAGAT $\left.[\mathrm{C}]_{14}\right)$ was performed for 3 cycles $\left(94^{\circ} \mathrm{C} / 20 \mathrm{~s}, 55^{\circ} \mathrm{C} / 30 \mathrm{~s}, 72^{\circ} \mathrm{C} /\right.$ $90 \mathrm{~s}$ ). The T7 primer ( $5^{\prime}$ GCCAGTGAATTGTAATACGAC) was then added for another 25 cycles $\left(94^{\circ} \mathrm{C} / 20 \mathrm{~s}, 60^{\circ} \mathrm{C} / 30 \mathrm{~s}, 72^{\circ} \mathrm{C} / 90 \mathrm{~s}\right)$. The resulting product was treated with proteinase $\mathrm{K}$, phenol/chloroform extracted, and ethanol precipitated. Each series of reactions also included normal human thymus RNA as an internal control, as well as reagent controls. Synthetic RNA was subsequently generated by transcription of the PCR product using T7 RNA polymerase reactions containing $[0.5 \mu \mathrm{M}]$ NTPs (Riboprobe System; Promega Biotech, Madison, WI).

$R P A$. The methodology of the multiprobe RPA has been previously detailed $(13,14)$. In brief, amplified synthetic RNA was transcribed in the presence of ${ }^{35}$ S $]$ UTP (Fig. 1), and an aliquot $(1,000$ $\mathrm{cpm}$ ) was incubated overnight with one of three labeled BV probe
TCR MRNA
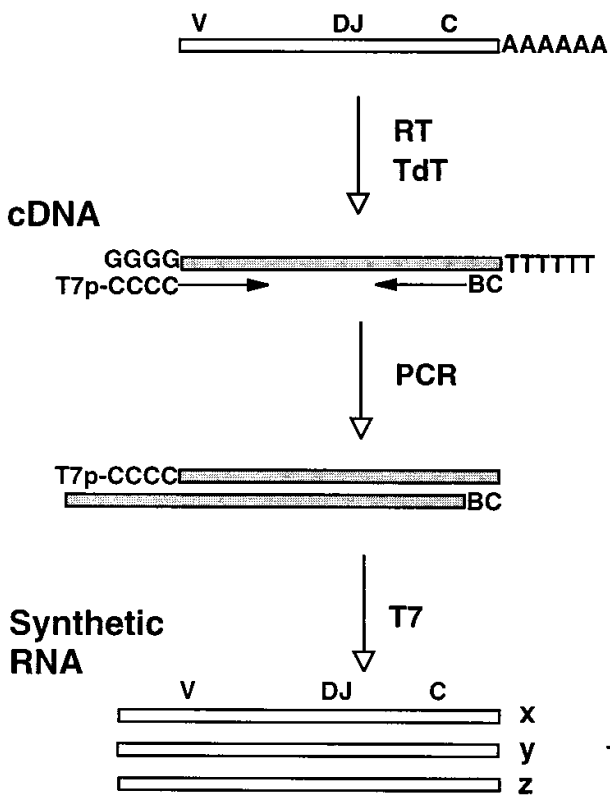

Autoradiograph

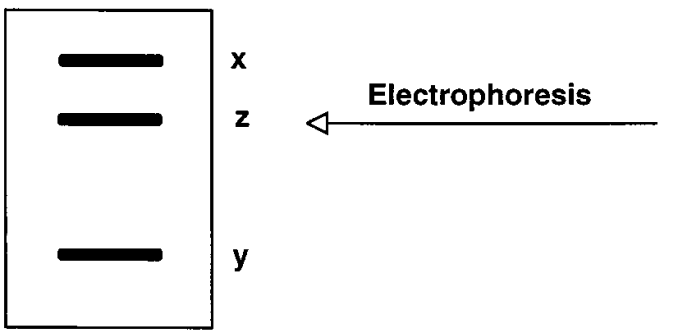

Plasmids

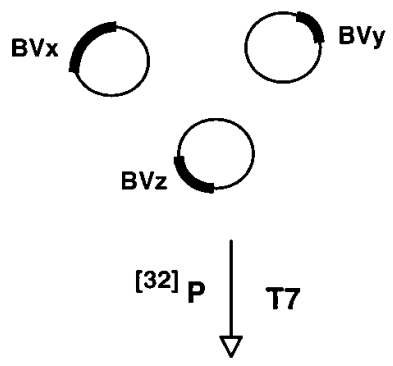

CRNA Figure 1. Schematic representation of methodology for quantitation of TCR gene expressions. cDNA is synthesized from mRNA using reverse transcriptase $(R T)$ and $5^{\prime}$ oligo-dG tails are added by terminal deoxynucleotidyl transferase $(T d T)$. The cDNA is amplified by PCR using an oligo-dC primer that incorporates a T7 RNA polymerase promoter region $(T 7 p)$, and a primer to the constant region of the TCR $\beta$-chain (BC). RNA is synthesized from the PCR product by T7 RNA polymerase. This RNA is hybridized to an excess of radiolabeled antisense probes transcribed from specific BV genes that have been cloned into plasmids. RNA duplexes (target RNA-probe) are protected from digestion by RNases, and resolved on acrylamide gels by electrophoresis. Measurement of $\beta$ radiation corresponding to particular $\mathrm{BV}$ bands yields relative levels of mRNA in the original specimen. 
sets $\left(1 \times 10^{6} \mathrm{cpm}\right)$ and labeled TCRBC probe $\left(1 \times 10^{5} \mathrm{cpm}\right)$. After digestion of unhybridized RNAs and ethanol precipitation, samples were electrophoresed on polyacrylamide sequencing gels for autoradiography and direct $\beta$ counting (Radioanalytical Imaging Apparatus; Ambis Systems, San Diego, CA). Results for each of the 27 BV gene probes were expressed as percentages of total measured BV. Specimens showing abnormal BV biases were run in duplicate and the results averaged.

Cloning and sequencing. Details of this methodology have been provided previously $(13,14)$. In brief, the gene sequence of interest was amplified by PCR using TCRBV-specific and BC primers. Products of this reaction were cloned (TA Cloning Kit; Invitrogen Corp., San Diego) and plasmid DNA was sequenced by PCR using the fmol Kit (Promega).

Statistics. Two-group comparisons of continuous or ordered data were analyzed by Mann-Whitney test. Nominal values were compared by Chi square. Correlation coefficients for continuous data were established by linear regression and Pearson product-moment correlation $(r)$. The Spearman rank correlation coefficient method $(s)$ was used to define correlations between continuous and ordered data. The normal range for individual TCRBV gene expressions was defined as the mean $\pm 3 \mathrm{SD}$ of mean values obtained from normal volunteers. Data are denoted as means \pm SE. Statistical significance was defined as $P<0.05$.

\section{Results}

Specimens. A total of 52 specimens were evaluated from 24 transplant recipients. Characteristics of the study populations are detailed in Table I. TCRBV analyses were also performed using PBL from 17 normal volunteers (mean age 39.8 \pm 3.2 ). Eleven OB and six NER recipients had concomitant BAL and PBL specimens.

Two or more serial PBL specimens were obtained in seven

Table I. Characteristics of Study Populations*

\begin{tabular}{|c|c|c|}
\hline & $\mathrm{OB}$ & NER \\
\hline$n$ & 16 & 9 \\
\hline age (yr) & $38.8 \pm 1.8$ & $38.2 \pm 3.7$ \\
\hline POD & $1395 \pm 262$ & $794 \pm 384$ \\
\hline $\mathrm{FEF}_{25-75}$ & $37.2 \pm 9.7$ & $80.9 \pm 17.3^{\ddagger}$ \\
\hline \multicolumn{3}{|l|}{ Transplantation } \\
\hline HL & 12 & 5 \\
\hline DL & 3 & 1 \\
\hline SL & 1 & 3 \\
\hline \multicolumn{3}{|l|}{ Specimens analyzed } \\
\hline PBL & 23 & 11 \\
\hline BAL & 12 & 6 \\
\hline \multicolumn{3}{|c|}{ PBL subpopulations (\%) } \\
\hline $\mathrm{CD}^{+} / \mathrm{CD}^{+}$ & $42 \pm 5$ & $36 \pm 9$ \\
\hline $\mathrm{CD}^{+} / \mathrm{CD}^{+}$ & $37 \pm 6$ & $34 \pm 6$ \\
\hline $\mathrm{CD} 4^{+} \mathrm{DR}^{+} / \mathrm{CD} 4^{+}$ & $13 \pm 6$ & $8 \pm 5$ \\
\hline $\mathrm{CD}^{+} \mathrm{DR}^{+} / \mathrm{CD}^{+}$ & $15 \pm 4$ & $5 \pm 2$ \\
\hline
\end{tabular}

* One recipient, initially with NER, had a subsequent evaluation when OB was diagnosed, and is included in both populations. POD: postoperative day; $\mathrm{FEF}_{25-75}$ : forced expiratory flow over $25-75 \%$ of vital capacity (as percentage of predicted); HL; combined heart-lung; DL: doublelung; SL: single lung. ${ }^{\ddagger} P<0.05$.
OB patients with elapsed intervals of $201 \pm 24$ d between collections. Serial PBL specimens were also available from two NER recipients with elapsed intervals of $239 \pm 2 \mathrm{~d}$. One subject is included in both NER and OB subpopulations, having had PBL specimens collected on post-transplant day 144, with normal lung biopsies, and $240 \mathrm{~d}$ later, at which time OB was diagnosed.

$T C R B V$ analyses of $P B L$. The correlation coefficient of TCRBV values between RPA performed using unamplified cellular RNA and duplicate determinations after PCR amplification was $0.87 \pm 0.04(n=10)$, thus demonstrating that differential PCR amplification did not occur. Reproducibility of the methodology was comparable, with $r=0.88 \pm 0.02$ between duplicate analyses of amplified specimens.

Intergroup comparisons of TCRBV repertoires showed that PBL profiles of OB recipients were highly biased compared to those of the NER (Figs. 2 and 3). 69 of 432 individual TCRBV values $(16 \%)$ among OB recipients were abnormally expanded, whereas 22 of $243(9 \%)$ determinations among NER fell above normal ranges $(P=0.016)$. All 16 OB and $8 / 9$ NER had one or more abnormal PBL TCRBV. The mean

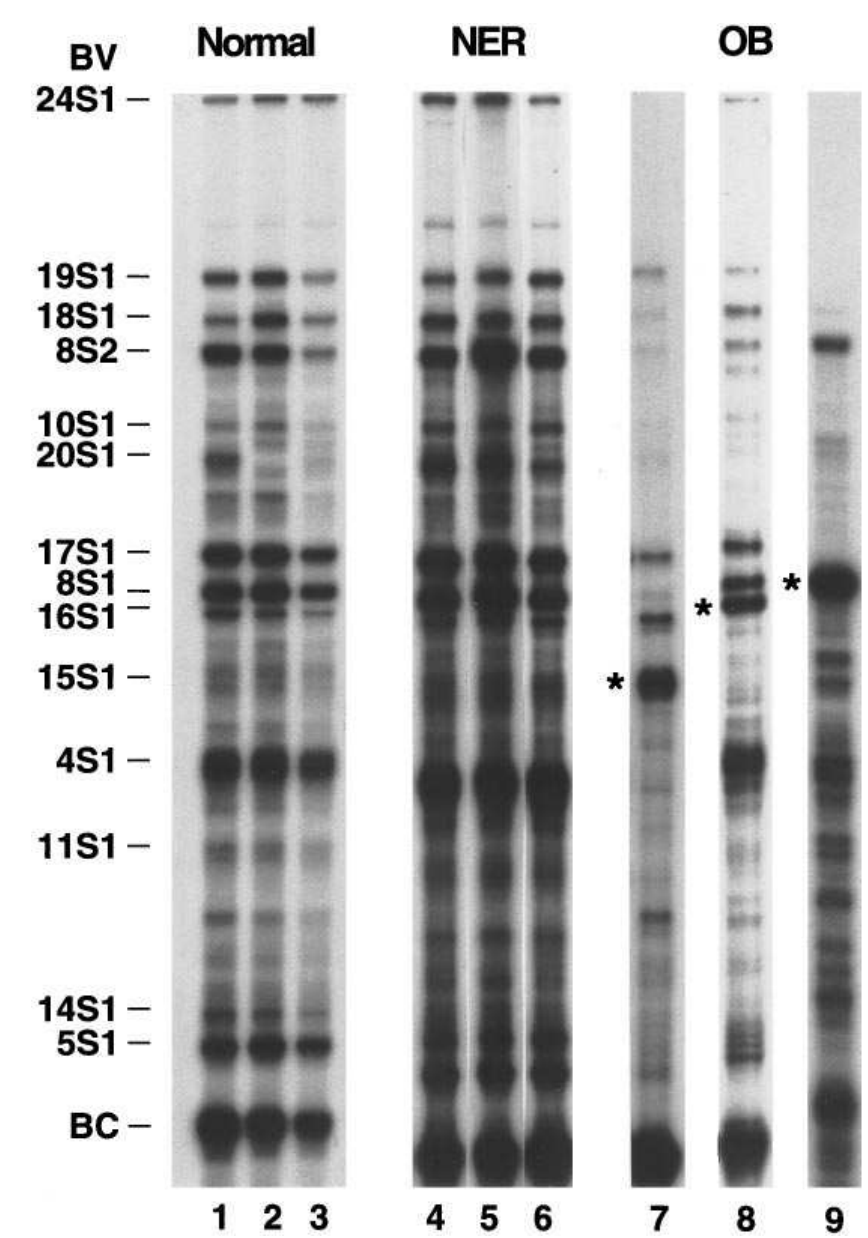

Figure 2. Composite autoradiograph of normal volunteer PBL, as well as PBL from lung transplants with NER or OB. Data are shown for BV genes incorporated into probe set one (of three). Whereas volunteer and NER profiles are normally distributed, abnormally expanded TCRBV ( $>3$ SD from mean normal values) among these OB are denoted by *. 


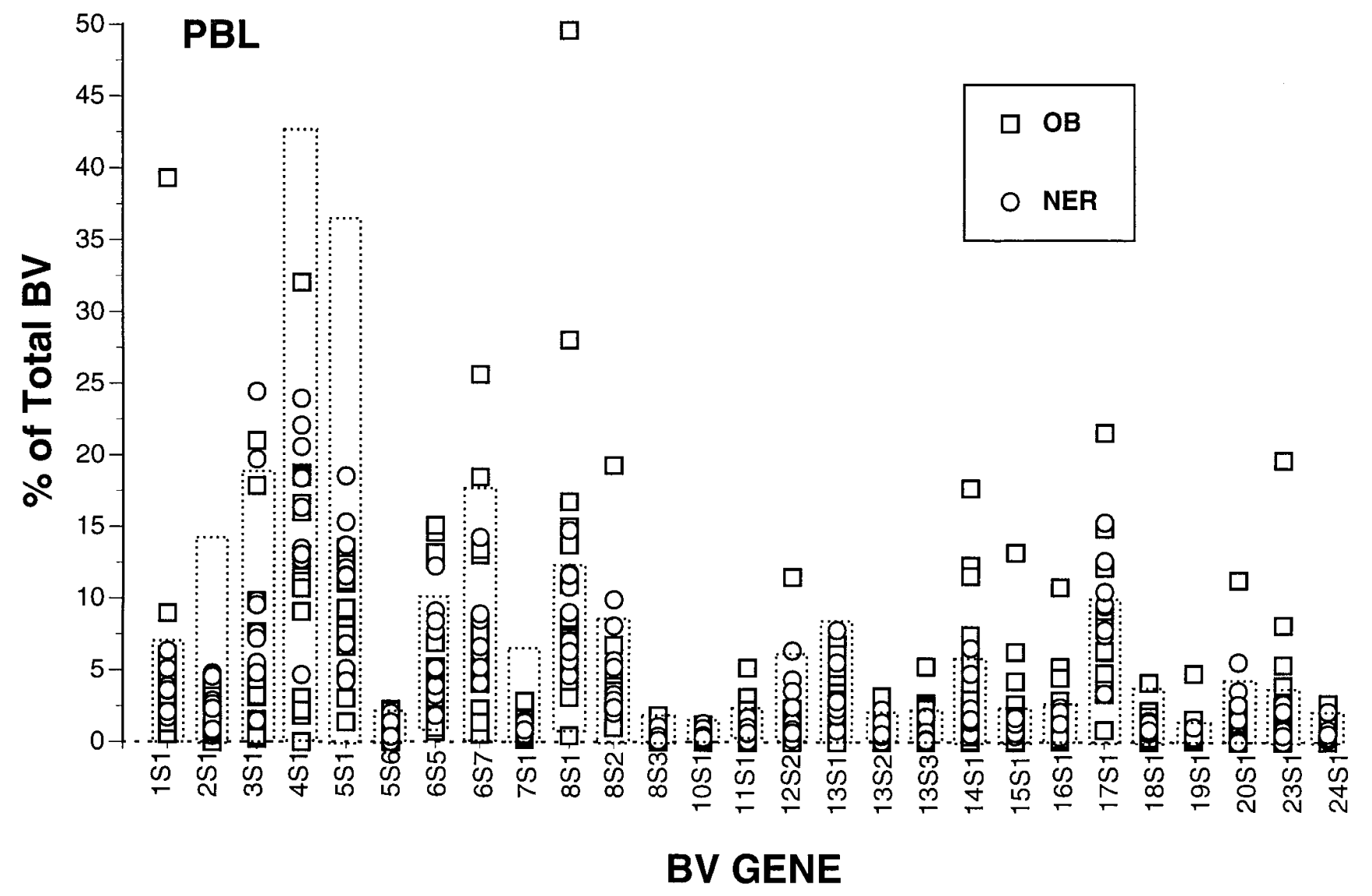

Figure 3. TCRBV gene expression levels in PBL of lung transplant recipients with NER or OB. Dotted-line boxes denote normal range ( \pm 3 SD from mean control values).

number of these skewed expressions per subject was $4.3 \pm 0.6$ among OB compared to $2.3 \pm 0.5$ for NER $(P=0.034)$.

More significantly, the magnitude of the PBL skewing was much greater in the $\mathrm{OB}$ recipients compared to those with NER. Mean coefficients of variation for values among the 27 individual PBL TCRBV genes were significantly greater in the OB (107.8 \pm 7.8$)$ compared to NER $(65.9 \pm 5.0)(P<<0.01)$. Values for abnormal TCRBV among OB recipients were $8.2 \pm 0.8 \mathrm{SD}$ from mean normal expressions, compared to $4.5 \pm 0.3 \mathrm{SD}$ for NER $(P<0.01)$ (Fig. 4$)$. The probability that OB recipients had one or more TCRBV expression levels $>7$ SD from respective normal values was $69 \%$. The specificity of these findings for presence of OB was $89 \%$. The positive and negative predictive values for presence of $\mathrm{OB}$ with findings of abnormal TCRBV levels $>7$ SD from mean controls were 92 and $61 \%$, respectively.

There were no apparent correlations between elapsed time since transplantation and the number of PBL TCRBV abnormalities $(r=0.27)$ or magnitudes of these abnormalities $(r=$ $0.03)$.

There appeared to be trends for preferential expansions of certain genes among PBL of OB recipients, although none of these differences reached statistical significance. For example, five of the OB recipients studied (31\%) had elevated levels of BV 8S1 among their PBL, whereas this finding was only present in one NER, and this subject subsequently developed OB.

$T C R B V$ analyses of BAL. Unlike PBL specimens, TCRBV profiles of BAL from both transplant recipient populations exhibited considerable degrees of skewing (Fig. 5). 44 of 297 (15\%) TCRBV from OB BAL were above normal values established from control PBL; 29/162 (18\%) BAL TCRBV among NER were similarly elevated. The absolute numbers of

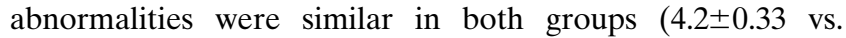
$4.8 \pm 0.4, \mathrm{OB}$ and NER, respectively), as were their magnitudes (7.8 \pm 0.8 vs. $6.6 \pm 0.9$ SD from mean normal, OB and NER, respectively). Dispersions of values were also similar with mean coefficients of variation $95.8 \pm 7.5$ among $\mathrm{OB}$ and $88.0 \pm 5.3$ in the NER.

In general, there was often considerable variability between PBL and BAL TCRBV profiles of individual subjects (Fig. 6). Only $35 \%$ of abnormal TCRBV in PBL were associated with expansions of corresponding BAL genes, and there were no differences in this regard between NER and OB recipients. Conversely, 29 and $12 \%$ of BAL expansions were associated with abnormalities in expression levels of the corresponding PBL genes among $\mathrm{OB}$ and NER, respectively $(P=$ 0.12). Correlation coefficients between BAL and PBL TCRBV repertoires were $0.62 \pm 0.07$ among NER, and $0.54 \pm 0.07$ among the OB.

Serial analyses. PBL TCRBV profiles of individual subjects tended to remain stable over the intervals studied here. Overall, $r$ of repeated PBL specimens was $0.80 \pm 0.03$ among NER, and $0.79 \pm 0.04$ among OB. There was a high degree of concordance between PBL repertoires of two NER who re- 


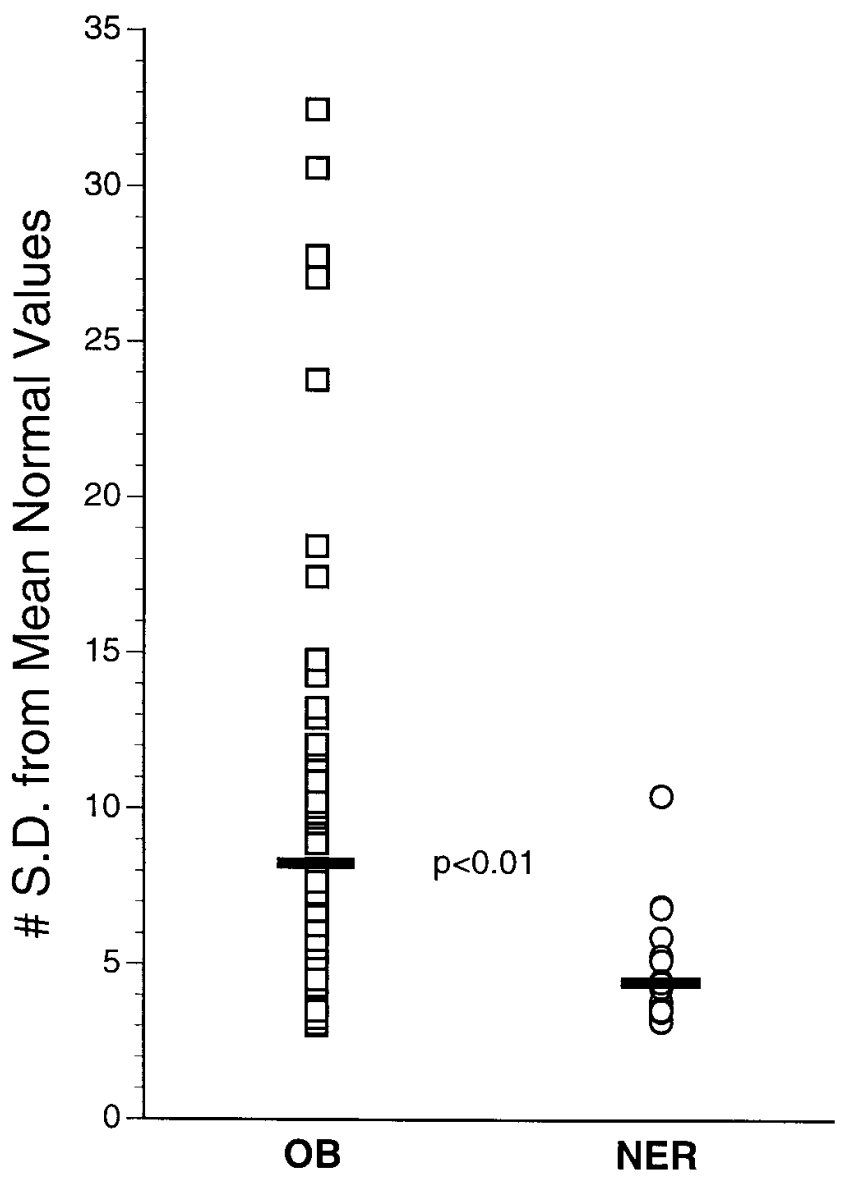

Figure 4. Magnitudes of TCRBV abnormalities (number of standard deviations from mean normal values) in peripheral blood specimens of transplant recipients with OB and NER. Mean values are denoted by horizontal bars.

ceived single lung transplants from a common donor $(r=$ 0.93), whereas BAL specimens from these subjects showed little similarity $(r=0.4)$. The $r$ between serial BAL profiles of a single $\mathrm{OB}$ recipient was 0.62 .

$T C R$ sequences. Junctional region sequences of selected expanded BV were determined using seven PBL and five BAL specimens from OB recipients. The presence of mono- or oligoclonality was readily established in all but one (Table II). The proportion of total TCRBV gene expression that derived from a limited number of clones was often quite striking. For instance, $72 \%$ of TCRBV expression in patient \#8 is attributable to proliferations of three clonal progenitors (bearing $\mathrm{BV}$ $1 \mathrm{~S} 1,5 \mathrm{~S} 3,15 \mathrm{~S} 1$, respectively), and $\sim 40 \%$ of total TCRBV in patient \#20 is due to expansions of a single 8S1 T cell.

The preferential expansions of BV 8S1 among PBL of OB recipients were examined in particular detail, but there was no apparent VDJ commonality between individual patients. In contrast, however, two patients shared a common VDJ sequence (BV20S1) in their BAL specimens. The possibility that these findings were due to cross contamination was remote given that more than a year elapsed between collection (and processing) of these specimens. Three MHC alleles were common to both recipients, whereas none of the shared alleles among their respective donors were allogeneic to both recipients (data not shown).

\section{Discussion}

These data demonstrate that TCRBV repertoires of circulating $\mathrm{T}$ cells among long-term survivors of lung transplantation are highly biased relative to those of healthy, normal controls. More importantly, both the number and magnitude of these $\mathrm{T}$ cell expansions are greater among recipients with obliterative bronchiolitis, compared to those with no evidence of rejection. Furthermore, these extreme TCRBV expansions are clonal or oligoclonal, i.e., progeny of one or a very few $\mathrm{T}$ cells that have been intensely stimulated to proliferate to a degree that approaches that of neoplastic lymphoid diseases. To our knowledge, these are the first data that have extensively, and by quantitative methodology, concurrently examined the TCRBV repertoires of blood and graft infiltrating lymphocytes among human transplant recipients with chronic allograft rejection. These findings offer insights into mechanisms of alloreactivity, and suggest the pathogenesis of chronic rejection involves specific, cognate interactions between $T$ cells and a surprisingly small number of immunodominant alloantigens.

The molecular basis of alloantigen recognition has long been the subject of intense interest $(7,8,15)$. As many as $1-5 \%$ of unprimed $\mathrm{T}$ cells may respond to an allogeneic haplotype in an in vitro mixed lymphocyte reaction, a reflection of engagements by lymphocytes bearing enormously diverse TCR (15, 16). Nonetheless, an increasing number of reports suggest that in vivo allogeneic responses may be mediated by much more limited, or biased, repertoires of alloreactive T cells. The presence of dominant, albeit uncharacterized, TCRBV gene rearrangements has previously been detected by Southern blots of $\mathrm{T}$ cell lines derived from biopsies of human renal and cardiac allografts (17-19). Restricted usage of TCRBV genes during acute rejection has also been reported in evaluations of graft infiltrating lymphocytes in animal models $(20,21)$.

Findings that the TCR repertoires of responders to allogeneic transplants are restricted may, at first glance, be difficult to reconcile with the known high precursor frequency of potential alloreactive cells. Among the most likely explanations, these TCR gene expression biases and clonal expansions may be a reflection of indirect alloantigen recognition, a putative mechanism of allograft recognition and rejection that has been a focus of several recent investigations (22-24). As demonstrated in various models (24-30), allogeneic peptides, including polymorphic sequences derived from donor $\mathrm{MHC}$, are capable of uptake by recipient antigen-presenting cells, and are subsequently presented to T cells with, in turn, evocation of alloantibody and cytotoxic immune responses. Of particular relevance given the present findings, indirect responses to allopeptide-self-MHC complexes appear to mimic those of other nominal peptide antigens (31-33) in that the precursor frequency of reactive T-cells is at least 100 -fold less than those that are directly engaged by in vitro MLR (i.e., direct alloantigen recognition). Accordingly, expansions of $\mathrm{T}$ cells mediating indirect responses to allopeptides tend to be focused or biased to a relatively small number that display the most effective TCR junctional region sequences $(29,30)$.

Demonstrations that significant alloresponses are attributable to a limited repertoire of $\mathrm{T}$ cells have clinically relevant implications. Most importantly, therapies that exploit the pres- 

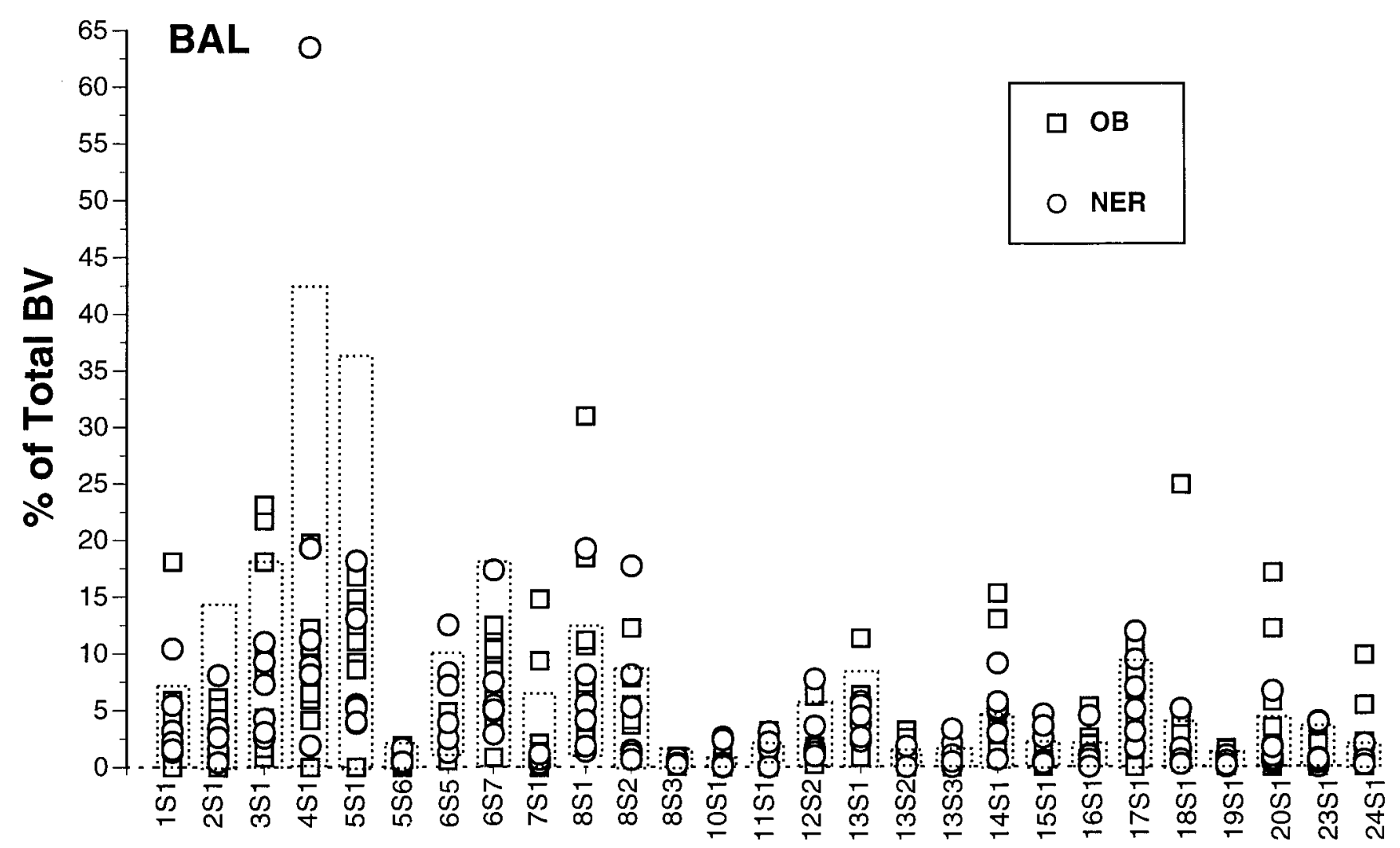

BV GENE

Figure 5. TCRBV gene expression levels in BAL of lung transplant recipients with NER or OB. Dotted-line boxes denote normal range $( \pm 3$ SD from mean control values).

ence of predominant alloreactive TCR phenotypes or otherwise interfere with specific TCR-allopeptide engagements could have considerable potential therapeutic utility $(9,10)$. Augmentation of conventional immunosuppressants (e.g., cyclosporine, OKT3, glucocorticosteroids) is associated with considerable risks of opportunistic infections and lymphomagenesis since $\mathrm{T}$ cell functions are globally depressed $(1,34)$ and is, in any case, of dubious therapeutic efficacy for chronic rejection $(2,3,35)$. As noted here, TCRBV profiles of expanded $\mathrm{T}$ cell clonotypes are likely to vary among individual recipients, a reflection of responses to unique combinations of allogeneic determinants. Nonetheless, appropriately tailored antiTCR modalities could be useful adjuncts that specifically abrogate allograft injury, while leaving the brunt of other useful immune responses intact $(9,21)$.

In addition, if distinctive TCRBV repertoires of individual recipients are temporally stable and the magnitude of their abnormalities are discriminative as suggested here, assays for perturbations of TCR profiles could conceivably have value for diagnosis and allograft surveillance. Finally, findings here that $\mathrm{T}$ cell populations of transplant recipients are largely constituted by a small number of expanded clones implies that many of the specificities of a normal TCR repertoire are present in much lower precursor frequencies. These "holes" in the repertoire may account to some degree for the increased risk of transplant recipients, especially those with chronic rejection, to develop opportunistic infections and other complications due to cellular immune abnormalities $(1,34,35)$. Ac- cordingly, it is possible that treatments which deplete dominant (alloreactive) clones may restore the overall frequencies of other populations that display beneficial specificities for microbial pathogens and tumor suppression.

In contrast to analyses of PBL, the TCRBV repertoires of BAL specimens are highly biased among both NER and OB, and may be less temporally stable. Furthermore, concomitant PBL and BAL of individual patients are often considerably disparate. Given that most, if not all, of the striking PBL and BAL expansions are oligoclonal (thus likely representing specific proliferative responses to a particular antigen), it is highly unlikely that the respective differences of $\mathrm{T}$ cell clonotypes can be merely attributed to preferential sequestrations. While definitive explanations for these findings cannot be established from the current data, it may be possible that discordances between individual PBL and BAL profiles are due to differential expression or density of immunogenic alloantigens among pulmonary airspace and vascular compartments. Discordances would also result if circulating $T$ cells that engage alloantigens in the vascular or perivascular spaces (and/or peripheral lymphatic organs) underwent preferential local expansions, and did not freely or completely transit into pulmonary airspaces. Finally, assessments of $\mathrm{T}$ cell responses in BAL could readily be confounded by local $\mathrm{T}$ cell responses to inhaled antigens or infectious antigens in the airspaces. Overt infections, as well as subclinical microbial colonizations, are frequent in the lower respiratory tract of lung transplant recipients $(1,34,35)$ and the resultant inflammatory response could have long-lasting 


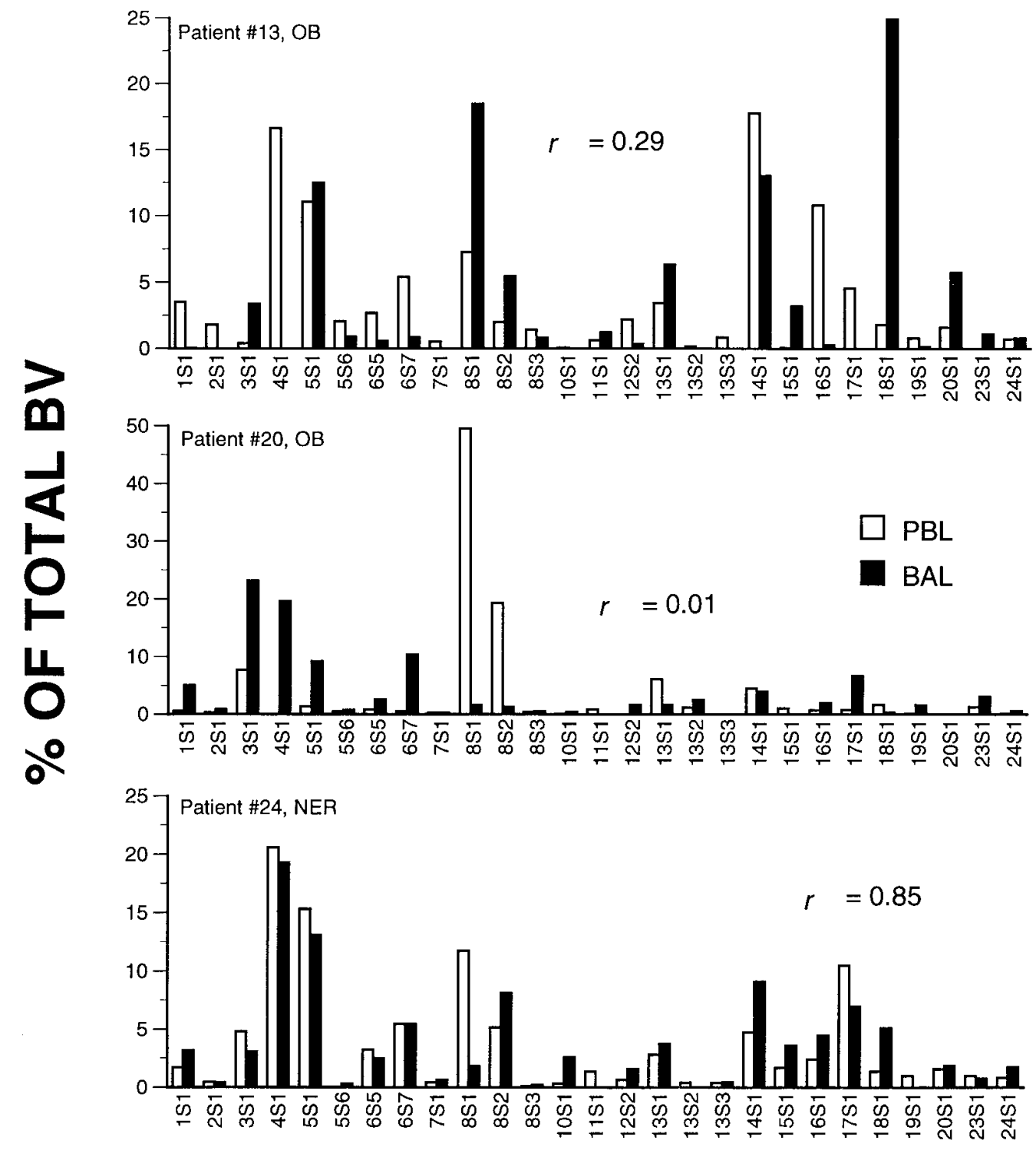

BV GENE
Figure 6. Concordances between paired $\mathrm{PBL}$ and $\mathrm{BAL}$ TCRBV repertoires varied widely among individuals. $P B L$, peripheral blood; $B A L$, bronchoalveolar lavage; $N E R$, no evidence of rejection; and $O B$, obliterative bronchiolitis. effects on TCR repertoires. The fortuitous finding here that two recipients shared identical junctional region sequences among their BAL is consistent with proliferations of $\mathrm{T}$ cells mediating responses to common, albeit unknown, infectious or environmental antigens presented in the context of shared MHC (36).

These data demonstrate that abnormal T cell clonal expansions are prevalent in the circulation and pulmonary airspaces of lung transplant recipients. These studies also showed that the $\mathrm{T}$ cell expansions are qualitatively and quantitatively exaggerated in the circulation of lung allograft recipients with $\mathrm{OB}$, thus strongly implying that cognate, TCR-mediated responses are an important component of this transplantation complication. In all probability, these focused proliferations are the result of repeated engagements, over protracted periods, between a finite diversity of alloantigens and highly selected $\mathrm{T}$ cells bearing unique, specific TCR. It is entirely probable that the NER patients also have detectable $T$ cell clones among their PBL and BAL. Indeed several published reports detail the presence of benign $\mathrm{T}$ cell clones in healthy normals (reviewed in reference 37). Nonetheless, none of these earlier studies, nor data accumulated in our laboratory in the course of this or other investigations $(38,39)$ have found clonal expansions in PBL that begin to approach the extent and magnitude of those described here in the OB patients. Overall, the present data are consistent with and extend recent in vitro and animal model studies that have suggested indirect alloantigen recognition plays an important role in the pathogenesis of chronic allograft rejection $(3,22-30)$. Definitive evaluation of this hypothesis will ultimately be possible by establishing the antigenic specificities of the expanded $\mathrm{T}$ cell clonotypes, and such studies are in progress.

\section{Acknowledgments}

This work was supported in part by National Institutes of Health grants T32GMO8172, 1KO8HL03016, AR31203, and AR39555. 
Table II. Clonal Proliferations in Lung Transplant Receipients with OB

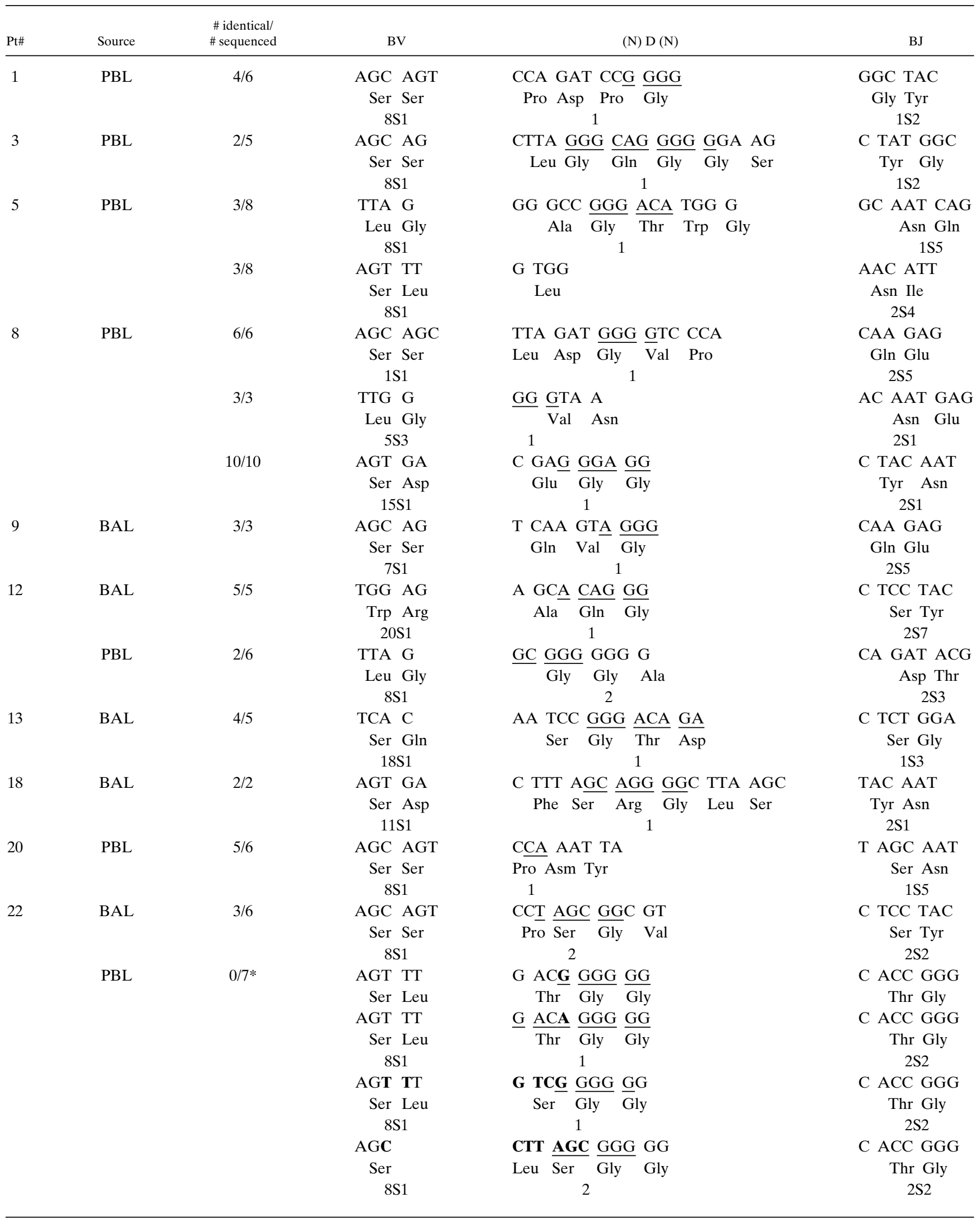

Patients designations (Pt) and specimen sources (PBL, BAL), numbers of sequences determined for each, and the numbers of identical (clonal) sequences found are denoted. Nucleotide sequences of terminal BV, diversity (D), and 5' regions of joining (BJ) genes are described, as well as the corresponding amino acid sequences. Identified D sequences are underlined and designated. *Although none of the seven sequences shared identical nucleotides, amino acid sequences were conserved among two pairs. Nucleotide differences between respective pairs are denoted in bold font. 


\section{References}

1. Duncan, S.R., I.L. Paradis, S. Similo, S.A. Yousem, P. Williams, W. Grugrich, J.P. Dauber, and B.P. Griffith. 1992. Sequelae of cytomegalovirus pulmonary infections in lung allograft recipients. Am. Rev. Resp. Dis. 146:1419-1425.

2. Theodore, J., V.A. Starnes, and N.J. Lewiston. 1990. Obliterative bronchiolitis. Clin. Chest. Med. 11:309-322.

3. Azuma, H., and N.L. Tilney. 1994. Chronic graft rejection. Curr. Opin. Immunol. 6:770-776.

4. Panzara, M.A., J.R. Oksenberg, and L. Steinman. 1992. The polymerase chain reaction for detection of T-cell antigen receptor expression. Curr. Opin. Immunol. 4:205-210

5. Theofilopoulos, A.N., R. Baccala, R. Gonzalez-Quintial, D.H. Kono, R.S. Balderas, R. MacPhee, S.R. Duncan, and M. Roglic. 1995. T-cell repertoires in health and disease. Ann. NY Acad. Sci. 756:53-65.

6. Theofilopoulos, A.N., R.S. Balderas, R. Baccala, and D.H. Kono. 1992. T-cell receptor genes in autoimmunity. Ann. NY Acad. Sci. 681:53-65.

7. Krensky, A.M., A. Weiss, G. Crabtree, M.M. Davis, and P. Parham. 1990. T-lymphocyte-antigen interactions in transplant rejection. N. Engl. J. Med. 322: 510-517.

8. Colvin, R.B. 1990. Cellular and molecular mechanisms of allograft rejection. Annu. Rev. Med. 41:361-375.

9. Adorini, L., J.C. Guery, G. Rodriguez-Tarduchy, and S. Trembleau. 1993. Selective immunosuppression. Immunol. Today. 14:285-289.

10. Gaur, A., and C.G. Fathman. 1994. Immunotherapeutic strategies directed at the trimolecular complex. Adv. Immunol. 56:219-265.

11. Yousem, S.A., G.J. Berry, E.M. Brunt, D. Chamberlain, R.H. Hruban, R.K. Sibley, S. Stewart, and H.D. Tazelaar. 1990. A working formulation for the standardization of nomenclature in the diagnosis of heart and lung rejection: lung rejection study group. J. Heart Transplant. 9:593-601.

12. Chomczynski, P., and N. Sacchi. 1987. Single-step method of RNA isolation by acid quanidium thiocyanate-phenol-chloroform extraction. Anal. Biochem. 162:156-159.

13. Plaza, A., D.H. Kono, and A.N. Theofilopoulos. 1991. New human V $\beta$ genes and polymorphic variants. J. Immunol. 147:4360-4365.

14. Kono, D.H., R. Baccala, R.S. Balderas, S.J. Kovac, P.W. Heald, R.I. Edelson, and A.N. Theofilopoulos. 1992. Application of a multiprobe RNase protection assay and junction sequences to define $\mathrm{V} \beta$ gene diversity in Sezary Syndrome. Am. J. Pathol. 140:823-830.

15. Warrens, A.N., G. Lombardi, and R.I. Lechler. 1994. MHC and alloreactivity. Transplant. Immunol. 2:103-107.

16. Matzinger, P., and M.H. Bevan. 1977. Hypothesis: why do so many lymphocytes respond to major histocompatibility antigens. Cell Immunol. 29:1-5.

17. Miceli, M.C., and O.J. Finn. 1989. T cell receptor $\beta$-chain selection in human allograft rejection. J. Immunol. 142:81-86.

18. Frisman, D.M., A.A. Hurwitz, W.T. Bennett, G.W. Dec, L.A. Boyle, J.T. Fallon, R.B. Colvin, and J.T. Kurnick. 1990. Clonal analysis of graft-infiltrating lymphocytes from renal and cardiac biopsies. Hum. Immunol. 28:208-215.

19. Hand, S.L., B.L. Hall, and O.J. Finn. 1990. T-cell receptor gene usage and expression in renal allograft-derived T-cell lines. Hum. Immunol. 28:82-95.

20. Shirwan, H., D. Chi, L. Makowa, and D.V. Cramer. 1993. Lymphocytes infiltrating rat cardiac allografts express a limited repertoire of T cell receptor $\beta$ genes. J. Immunol. 151:5228-5238.

21. Goss, J.A., R. Pyo, M.W. Glye, J.M. Connolly, and T.H. Hansen. 1993. Major histocompatibility complex-specific prolongation of murine skin and cardiac allograft survival after in vivo depletion of $\mathrm{V} \beta+\mathrm{T}$ cells. J. Exp. Med. 177: 35-44.
22. Krensky, A.M., and C. Clayberger. 1994. The induction of tolerance to alloantigens using HLA-base synthetic peptides. Curr. Opin. Immunol. 6:791796.

23. Shoskes, D.A., and K.J. Wood. 1994. Indirect presentation of MHC antigens in transplantation. Immunol. Today. 15:32-38.

24. Bradley, J.A., A.M. Mowat, and E.M. Bolton. 1992. Processed MHC class I alloantigens as the stimulus for CD4+ T-cell dependent antibody-mediated graft rejection. Immunol. Today. 13:434-438.

25. Benchelou, G., P.A. Takizawa, C.A. Olson, M. McMillin, and E.E. Sercarz. 1992. Donor major histocompatibility complex (MHC) peptides are presented by recipient MHC molecules during graft rejection. J. Exp. Med. 175 305-308.

26. Lee, R.S., M.J. Grusby, L.H. Glimcher, H.J. Winn, and H. Auchincloss. 1994. Indirect recognition by helper cells can induce donor-specific cytotoxic T lymphocytes in vivo. J. Exp. Med. 179:865-872.

27. Fangman, J.R., R. Dalchau, and J.W. Fabre. 1992. Rejection of skin allografts by indirect allorecognition of donor class I major histocompatibility complex peptides. J. Exp. Med. 175:1521-1529.

28. Dalchau, R., J.R. Fangman, and J.W. Fabre. 1992. Allorecognition of isolated, denatured chains of class I and class II major histocompatibility complex molecules: evidence for an important role for indirect allorecognition in transplantation. Eur. J. Immunol. 22:669-677.

29. Liu, Z., Y.K. Sun, Y.P. Xi, A. Faffei, E.F. Reed, P. Harris, and N. SuciuFoca. 1993. Contribution of direct and indirect recognition pathways to $\mathrm{T}$ cell alloreactivity. J. Exp. Med. 177:1643-1650.

30. Liu, Z., Y.K. Sun, Y.P. Xi, B. Hong, P. Harris, E.F. Reed, and N. SuciuFoca. 1993. Limited usage of $\mathrm{T}$ cell receptor $\mathrm{V} \beta$ genes by allopeptide-specific $\mathrm{T}$ cells. J. Immunol. 150:3180-3186.

31. Hedrick, S.M., I. Engel, D.L. McElligott, P.J. Fink, M.L. Hsu, D. Hansburg, and L.A. Matis. 1988. Selection of amino acid sequences in the beta chain of the T cell antigen receptor. Science (Wash. DC). 239:1541-1544.

32. McHeyzer-Williams, M.G., and M.M. Davis. 1995. Antigen-specific development of primary and memory T-cells. Science (Wash. DC). 268:106-111.

33. Heber-Katz, E., and H. Acha-Orbea. 1989. The V-region disease hypothesis: evidence from autoimmune encephalomyelitis. Immunol. Today. 10: 164-169.

34. Duncan, S.R. 1993. Pulmonary complications of transplantation. In Current Pulmonology, Vol. 14. D.F. Tierney, editor. Mosby: Year Book Inc., St. Louis. 199-241.

35. Burke, C.M., J. Theodore, K.D. Dawkins, S.A. Yousem, N. Blank, M.E. Billingham, A. Van Kessel, S.A. Jamieson, P.E. Oyer, J.C. Baldwin, et al. 1984. Post-transplant obliterative bronchiolitis and other late lung sequelae in human heart-lung transplantation. Chest. 86:824-829.

36. Argaet, V.P., C.W. Schmidt, S.R. Burrows, S.L. Silins, M.G. Kurilla, D.L. Doolan, A. Suhrbier, D.J. Moss, E. Kieff, T.B. Sculley, and I.S. Misko 1994. Dominant selection of an invariant $\mathrm{T}$ cell antigen receptor in response to persistent infection by Epstein-Barr virus. J. Exp. Med. 180:2335-2340.

37. Morely, J.K., F.M. Batliwalla, R. Hingorani, and P.K. Gregersen. 1995. Oligoclonal CD8+ T cells are preferentially expanded in the CD57+ subset. $J$. Immunol. 154:6182-6190.

38. Gonzalez-Quintial, R., R. Baccala, R.M. Pope, and A.N. Theofilopoulos. 1996. Identification of clonally-expanded T cells in rheumatoid arthritis using a sequence enrichment nuclease assay. J. Clin. Invest. 97:1335-1343.

39. Duncan, S.R., K.W. Pekny, M. Roglic, D.J. Elias, and A.N. Theofilopoulos. 1995. T cell $\mathrm{V} \beta$ repertoire biases and clonal proliferation in malignant pleural effusions. Am. J. Respir. Crit. Care Med. 151:A212. (Abstr.) 